\title{
Overexpression of dendritic cell-specific intercellular adhesion molecule-3-grabbing nonintegrin-related protein in cervical cancer and correlation with squamous cell carcinoma antigen
}

\author{
XIANGDONG WANG ${ }^{1}$, YANGMEI JIANG ${ }^{2}$, MENGLANG YUAN ${ }^{1}$, CHUNLIN CHEN $^{1}$, \\ $\mathrm{KEYONG}_{\mathrm{WANG}}{ }^{3}$, QIANSHI ZHANG ${ }^{1}$, YUNFEI ZUO ${ }^{3}$ and SHUANGYI REN ${ }^{1}$ \\ ${ }^{1}$ Department of General Surgery, The Second Affiliated Hospital of Dalian Medical University, Dalian, Liaoning 116023; \\ ${ }^{2}$ Department of Clinical Laboratory, The First Affiliated Hospital of Dalian Medical University, Dalian, Liaoning 116011; \\ ${ }^{3}$ Department of Clinical Biochemistry, College of Laboratory Diagnostic Medicine, \\ Dalian Medical University, Dalian, Liaoning 116044, P.R. China
}

Received February 15, 2016; Accepted January 17, 2017

DOI: $10.3892 / \mathrm{ol} .2017 .6508$

\begin{abstract}
Dendritic cell-specific intercellular adhesion molecule-3-grabbing nonintegrin-related protein (DC-SIGNR) is a type II transmembrane protein that has been reported to bind to various pathogens and participate in immunoregulation and tumorigenesis. However, further research is required to investigate whether the level of DC-SIGNR and cervical cancer are associated. The present study aimed to explore the clinical diagnostic significance of DC-SIGNR in cervical cancer. Immunohistochemical staining of DC-SIGNR was performed in samples from 25 patients with early stage cervical cancer, 14 patients with cervical intraepithelial neoplasia (CIN) and cervical polyp samples from 15 individuals. DC-SIGNR expression in cervical cancer tissue was significantly higher compared with that in CIN and cervical polyp tissue $(\mathrm{P}=0.0184$ and $\mathrm{P}=0.0236$, respectively). However, there was no significant difference in DC-SIGNR expression between CIN and cervical polyp tissue $(\mathrm{P}=0.8103)$. Additionally, the serum DC-SIGNR levels in 84 cervical cancer patients and 69 healthy female individuals were measured using an ELISA. Serum (s)DC-SIGNR levels were significantly higher in cervical cancer patients compared with healthy female individuals $(\mathrm{P}<0.0001)$. A
\end{abstract}

Correspondence to: Dr Shuangyi Ren, Department of General Surgery, The Second Affiliated Hospital of Dalian Medical University, 467 Zhongshan Road, Dalian, Liaoning 116023, P.R. China

E-mail: rsydl@aliyun.com

Professor Yunfei Zuo, Department of Clinical Biochemistry, College of Laboratory Diagnostic Medicine, Dalian Medical University, 9 South Road West, Dalian, Liaoning 116044, P.R. China

E-mail: zuf04112002@dlmedu.edu.cn

Key words: dendritic cell-specific intercellular adhesion molecule-3-grabbing nonintegrin-related protein, cervical cancer, expression, correlation, squamous cell carcinoma antigen
sDC-SIGNR level of $93.7 \mathrm{ng} / \mathrm{ml}$ was revealed by receiver operating characteristic curve analysis to predict the presence of cervical cancer with $69.57 \%$ sensitivity and $66.67 \%$ specificity (area under the curve, 0.6989; $\mathrm{P}<0.0001$ ). Levels of sDC-SIGNR in cervical cancer patients were also correlated with serum levels of squamous cell carcinoma antigen $(\mathrm{r}=0.2583 ; \mathrm{P}=0.0348)$. The results of the present study demonstrate that DC-SIGNR is overexpressed in cervical cancer tissue, and suggest that DC-SIGNR could serve as a biomarker for the early diagnosis of cervical cancer. Nevertheless, further studies are required to demonstrate what role DC-SIGNR serves in cervical cancer.

\section{Introduction}

Cervical cancer is the second most common malignancy in women (1) and has a significant impact on their health and lives (2). According to the World Health Organization, 266,000 women worldwide die of cervical cancer annually. Cervical cancer is the most common cancer among women in 45 different countries; these include countries in sub-Saharan Africa, Asia, including India, and certain Central and South American countries (3). A variety of factors contribute to cervical cancer occurrence and development; for example, the high-risk human papilloma virus $(4,5)$. Cervical cancer susceptibility is also increased by host genetic factors (6). Additionally, there are other biological molecules that are correlated with the risk of cervical cancer, such as forkhead box protein P3 (7).

C-type lectins, a large superfamily of proteins, have been reported to serve important roles in immunity, cell death and tumorigenesis (8-11). Dendritic cell-specific intercellular adhesion molecule-grabbing nonintegrin-related protein (DC-SIGN) is a membrane-bound member of the C-type lectin superfamily that is expressed in dendritic cells, monocyte-derived dendritic cells and specialized macrophages in vitro (12). The genes encoding DC-SIGN and its homologue DC-SIGN-related protein (DC-SIGNR) are located on human chromosome 19p13.3, and belong to a subfamily in the lectin gene cluster along with cluster of differentiation (CD)23 and 
liver and lymph node sinusoidal endothelial cell C-type lectin (LSECtin) (13-15). DC-SIGN is also expressed in the endothelial cells of the hepatic sinusoid and lymphatic sinus (16). DC-SIGN expression is associated with colorectal cancer (17) and non-Hodgkin's lymphoma (NHL) (18); thus DC-SIGN may be useful in a clinical setting (18).

Since DC-SIGNR and DC-SIGN share a 77\% structural similarity (19), their function may be similar. DC-SIGNR is highly expressed in endothelial cells in the placenta, liver and lymph nodes (20), but is expressed at lower levels in NHL and lung cancer $(18,21)$. In addition, a previous study reported that serum concentrations of DC-SIGNR were higher in brain metastatic compared with in non-metastatic lung cancer (21). However, an association between DC-SIGNR and cervical cancer has not been reported, to the best of our knowledge. In the present study, the tissue expression of DC-SIGNR and levels of serum (s)DC-SIGNR in patients with cervical cancer were investigated in order to determine whether sDC-SIGNR could be used as biomarker for the detection of cervical cancer. Additionally, the association between the expression of DC-SIGNR and squamous cell carcinoma antigen (SCC-Ag), the serum marker typically used for the clinical monitoring of cervical cancer, was measured (22).

\section{Materials and methods}

Clinical samples. Cervical tissue samples from 25 patients with cervical cancer, 14 patients with cervical intraepithelial neoplasia (CIN) and 15 patients with cervical polyps were obtained from the Second Affiliated Hospital of Dalian Medical University (Dailan, China) between July 2007 and September 2015. Additionally, 10 lymph node tissue samples from healthy individuals were obtained from the Second Affiliated Hospital of Dalian Medical University between August 2009 and May 2015. The lymph node tissue was used as a positive control for DC-SIGNR expression, while the cervical polyp tissue was used as a negative control. The clinical data for these subjects are summarized in Table I.

Serum samples from 84 patients with cervical cancer were obtained between November 2012 and November 2015 from the Second Affiliated Hospital of Dalian Medical University and stored at $-80^{\circ} \mathrm{C}$ until required. The mean age of the 84 patients was 50.38 years old and the range was 17-74 years. Clinical staging of cervical cancer patients was performed according to the International Federation of Gynecology and Obstetrics stage (23). Clinical parameters including age, clinical stage, concentrations of lactate dehydrogenase (LDH), alkaline phosphatase (ALP), SCC-Ag, serum carcinoembryonic antigen (CEA), carbohydrate antigens (CAs)-199, -153 and -125, and T cell subsets were acquired from hospitalization records. The data are illustrated in Table II. The control group was composed of 69 healthy blood donor volunteers, which were obtained from the Second Affiliated Hospital of Dalian Medical University between June 2013 and September 2015. The mean age of the control group volunteers was 52 years old, with a range of 23-74 years. Each healthy volunteer was submitted to a routine physical examination, and all of the results were in the normal range. Patients and healthy individuals with severe infections, known allergies and cachexia were excluded from the present study. The present study was approved by the Research Ethics Committee of Dalian Medical University and all participants provided written informed consent prior to enrollment.

Immunohistochemistry. Sections from paraffin-embedded blocks of each tissue sample were incubated at $60^{\circ} \mathrm{C}$ for $30 \mathrm{~min}$. The paraffin was removed with xylene and the samples were rehydrated in a graded ethanol series. Tissue sections (5 $\mu \mathrm{m}$ thick) were washed with PBS and endogenous peroxidase activity was blocked with $3 \%$ hydrogen peroxide diluted in double-distilled water for $10 \mathrm{~min}$ at room temperature. Antigen retrieval was performed by heating the slides in $0.01 \mathrm{~mol} / \mathrm{l}$ citrate-buffered solution ( $\mathrm{pH}$ 6.0) for $20 \mathrm{~min}$ in a microwave oven. Following antigen retrieval, sections were washed with PBS and blocked with goat serum (ZSGB-BIO, Beijing, China) for $20 \mathrm{~min}$ at the room temperature. An anti-DC-SIGNR rabbit polyclonal antibody (cat. no. ab169783; dilution, 1:150; Abcam, Cambridge, UK) was incubated with sections overnight at $4^{\circ} \mathrm{C}$. The next day, sections were washed 3 times in PBS ( $5 \mathrm{~min} /$ wash). Then, the sections were incubated for $1 \mathrm{~h}$ at $37^{\circ} \mathrm{C}$ with a horseradish peroxidase-labeled anti-rabbit secondary antibody $(1: 1,000)$ and washed 3 times in PBS. The sections were developed by applying 3,3'-diaminobenzidine tetrahydrochloride (ZSGB-BIO) to the sections and then counterstaining with hematoxylin. An Olympus BX51 microscope (Olympus Corporation, Tokyo, Japan) was used to capture images of all sections. The mean density of the DC-SIGNR expression was obtained using Image-Pro Plus software (version 6.0; Media Cybernetics, Inc., Rockville, MD, USA).

ELISA. An anti-DC-SIGNR goat polyclonal antibody (cat. no. sc-17261; Santa Cruz Biotechnology, Inc., Dallas, TX, USA) was used at a final concentration of $0.27 \mu \mathrm{g} / \mathrm{ml}$ to coat 96 -well microplates before incubating the plates overnight at $4^{\circ} \mathrm{C}$. The next day, the plates were washed 3 times with PBS containing $0.05 \%$ Tween-20 (PBST; pH 7.4), and the wells were blocked with $5 \%$ bovine serum albumin (cat. no. Roche1073507801; BIOSHSARP, Inc., Hefei, China) at $37^{\circ} \mathrm{C}$ for $1 \mathrm{~h}$. Following 3 washes with PBST, $100 \mu 1$ of serum from patients with cervical cancer and healthy individuals were added to the wells, using $100 \mu \mathrm{l}$ PBS as a negative control. Each plate was incubated at $37^{\circ} \mathrm{C}$ for $1 \mathrm{~h}$, and then the wells were washed 3 times with PBST before adding $100 \mu \mathrm{l}$ anti-DC-SIGNR mouse monoclonal antibody (cat. no. MAB16211; R\&D Systems, Inc., Minneapolis, MN, USA), diluted to a concentration of $0.5 \mu \mathrm{g} / \mathrm{ml}$, to each well. The plates were incubated at $37^{\circ} \mathrm{C}$ for $1.5 \mathrm{~h}$, and then the wells were washed 3 times with PBST. Following the PBS wash, $100 \mu \mathrm{l}$ of a peroxidase-conjugated goat anti-mouse antibody (cat. no. ZB-2305; dilution, 1:1,500; ZSGB-BIO) was added and the plates were incubated for $60 \mathrm{~min}$ at $37^{\circ} \mathrm{C}$. The plates were washed 3 times with PBST and 3,3',5,5'-tetramethylbenzidine (Tiangen Biotech Co., Ltd., Beijing, China) was added to each well prior to incubation at $37^{\circ} \mathrm{C}$ for $30 \mathrm{~min}$. Finally, $2 \mathrm{~mol} / \mathrm{H} \mathrm{H}_{2} \mathrm{SO}_{4}$ was added to stop the reaction. The optical density (OD) was measured on a microplate reader at a wavelength of $450 \mathrm{~nm}$. The sDC-SIGNR concentration in each sample was calculated by comparing the OD values with a standard curve. 
Table I. Clinical data of patients with cervical cancer, cervical intraepithelial neoplasia and cervical polyps whose tissue samples were included in the immunohistochemical analysis.

\begin{tabular}{|c|c|c|c|}
\hline Clinical data & Cervical cancer $(\mathrm{n}=25), \mathrm{n}(\%)$ & CIN (n=14), n (\%) & Cervical polyp $(n=15), n(\%)$ \\
\hline \multicolumn{4}{|l|}{ Age } \\
\hline$\geq 50$ & $15(60)$ & $4(29)$ & $6(40)$ \\
\hline$<50$ & $10(40)$ & $10(71)$ & $9(60)$ \\
\hline \multicolumn{4}{|l|}{ SCC-Ag (ng/ml) } \\
\hline$\geq 2.5$ & $10(40)$ & $0(0)$ & $0(0)$ \\
\hline$<2.5$ & $6(24)$ & $5(36)$ & $1(7)$ \\
\hline No data available & $9(36)$ & $9(64)$ & $14(93)$ \\
\hline \multicolumn{4}{|l|}{ Mean density } \\
\hline$\geq 0.000124498$ & $23(92)$ & $9(64)$ & $9(60)$ \\
\hline$<0.000124498$ & $2(8)$ & $5(36)$ & $6(40)$ \\
\hline
\end{tabular}

The results indicate the number of patients for whom data were available. CIN, cervical intraepithelial neoplasia; SCC-Ag, squamous cell carcinoma antigen.

Table II. sDC-SIGNR levels according to age, cancer stage and levels of serum SCC-Ag.

\begin{tabular}{lcc}
\hline Clinical data & No. of patients $(\%)$ & sDC-SIGNR $(\mathrm{ng} / \mathrm{ml})$, median (range) $^{P_{\text {value }}}$ \\
\hline $\begin{array}{l}\text { Age }(\mathrm{n}=84) \\
\geq 50\end{array}$ & $45(54)$ & $115.7(51.15-769.5)$ \\
$<50$ & $39(46)$ & $118.0(29.64-247.2)$ \\
Cancer stage $(\mathrm{n}=82)$ & & \\
I-II & $60(73)$ & 9.7944 \\
III-IV & $22(27)$ & $99.3(42.27-225.0)$ \\
SCC-Ag $(\mathrm{n}=54)$ & & $148.3(42.27-378.9)$ \\
$\geq 2.5$ & $16(30)$ & $105.4(29.64-271.3)$ \\
$<2.5$ & $38(70)$ & 0.0336 \\
\hline
\end{tabular}

${ }^{\mathrm{a}}$ Mann-Whitney U test. The results indicate the number of patients for whom data were available. sDC-SIGNR, serum dendritic cell-specific intercellular adhesion molecule-3-grabbing nonintegrin-related protein; SCC-Ag, squamous cell carcinoma antigen.

Statistical analysis. A non-parametric Mann-Whitney U test was used to determine the statistical significance between groups in the immunohistochemical and ELISA analyses. A non-parametric Spearman's rank correlation coefficient test was performed to measure the correlation of sDC-SIGNR with SCC-Ag levels and other clinical parameters. Receiver operating characteristic (ROC) curve analysis was applied to assess the diagnostic value of sDC-SIGNR in cervical cancer. All statistical analyses were performed using GraphPad Prism 5 software (version 5; GraphPad Software, Inc., La Jolla, CA, USA).

\section{Results}

DC-SIGNR mean density is significantly increased in cervical cancer tissue compared with CIN and cervical polyp tissue. Immunohistochemical staining was used to explore whether DC-SIGNR is overexpressed in cervical cancer (Fig. 1). Previous studies have demonstrated that DC-SIGNR is expressed in normal lymph nodes (24), therefore normal lymph node tissue was included as a positive control (Fig. 1A). Staining of cervical tissue samples from 25 patients with cervical cancer, 14 patients with CIN and 15 patients with cervical polyps revealed that DC-SIGNR was overexpressed in cervical cancer tissue (Fig. 1C). The mean density of DC-SIGNR in cervical cancer tissue was significantly higher compared with CIN and cervical polyp tissue $(\mathrm{P}=0.0184$ and $\mathrm{P}=0.0236$, respectively; Fig. $1 \mathrm{~F}$ ); however, there was no significant difference between the mean density of DC-SIGNR expression in CIN and cervical polyp tissue $(\mathrm{P}=0.8103$; Fig. 1F). Additionally, the mean density of DC-SIGNR expression positively correlated with serum levels of SCC-Ag ( $\mathrm{r}=0.5143 ; \mathrm{P}=0.0203$; Fig. 2A). Furthermore, the mean density of DC-SIGNR was significantly increased in samples with serum SCC-Ag levels higher compared with the normal range $(\mathrm{P}=0.0433$; Fig. 2B). However, there was no correlation between serum LDH (Fig. 2C and D), ALP (Fig. 2E and F), or CD3, 4, and 8 levels (data not shown) and the mean density of 

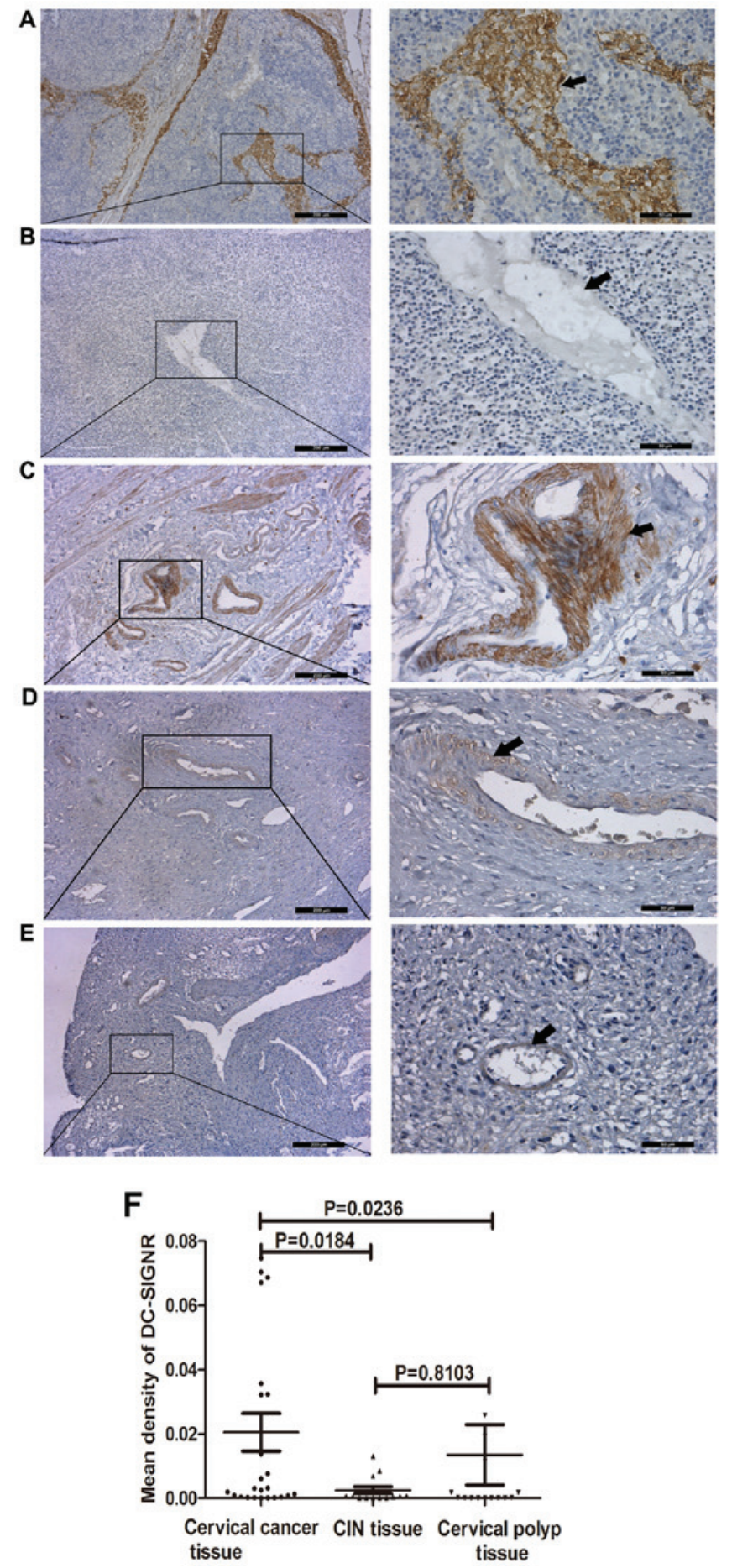

Figure 1. Immunohistochemical analysis of DC-SIGNR in cervical cancer, CIN and cervical polyp tissue samples. DC-SIGNR staining in (A) lymphatic endothelial cells (positive control), (B) normal lymph nodes without the addition of anti-DC-SIGNR (negative control), (C) cervical cancer tissue, (D) CIN tissue and (E) cervical polyp tissue. The arrows indicate positive staining. (F) A scatter plot of the mean density of DC-SIGNR in samples from 25 patients with cervical cancer, 14 patients with CIN and 15 patients with cervical polyps. Each dot represents the mean density of each sample. Data are presented as the mean \pm standard deviation. DC-SIGNR, dendritic cell-specific intercellular adhesion molecule-3-grabbing nonintegrin-related protein; CIN, cervical intraepithelial neoplasia.

DC-SIGNR. There was no significant difference between the mean density of DC-SIGNR in cervical cancer samples that were well differentiated compared with samples that exhibited low differentiation ( $\mathrm{P}=0.9650$; Fig. 3A) (25). Additionally, there was no significant difference between the mean density of DC-SIGNR in cervical cancer tissue from patients who were $\geq 50$ years old and patients who were $<50$ years old $(\mathrm{P}=0.6229$; Fig. 3B).

Levels of SDC-SIGNR are significantly higher in patients with cervical cancer compared with healthy individuals. Using an ELISA, the sDC-SIGNR levels of 84 patients with cervical cancer and 69 healthy individuals were compared. Levels of sDC-SIGNR in patients with cervical cancer were significantly higher compared with those in healthy individuals $(\mathrm{P}<0.0001$; Fig. $4 \mathrm{~A})$. Concentrations of sDC-SIGNR ranged from 29.64-769.5 $\mathrm{ng} / \mathrm{ml}$ (mean, $132.1 \mathrm{ng} / \mathrm{ml}$ ) in patients with cervical cancer and from 16.76-515.8 ng/ml (mean, $96.28 \mathrm{ng} / \mathrm{ml}$ ) in healthy individuals. A ROC curve is frequently used to evaluate the power of a novel serum marker to predict the presence of tumors. In the present study, a cut-off value of $93.7 \mathrm{ng} / \mathrm{ml}$ for sDC-SIGNR was identified to predict the presence of cervical cancer with $69.57 \%$ sensitivity and $66.67 \%$ specificity $(\mathrm{P}<0.0001$; Fig. 4B).

Levels of SDC-SIGNR are higher in stage I and II cervical cancer compared with stage III and IV cervical cancer. Excluding 2 patients with CIN III, levels of sDC-SIGNR were compared between 60 patients with stage I-II and 22 patients with III-IV cervical cancer, where stages I-II correspond to earlier stages of disease progression and would therefore have a better prognosis. Levels of sDC-SIGNR in patients with stage I and II cervical cancer were significantly higher than in patients with stage III and IV cervical cancer $(\mathrm{P}=0.0336$; Fig. 4C). There was no statistical significance between sDC-SIGNR levels in stage III and IV patients compared with healthy individuals $(\mathrm{P}=0.0759$; Fig. $4 \mathrm{C})$. These results suggest that sDC-SIGNR could be used as a biomarker for the early diagnosis of cervical cancer. However, there was no significant difference in sDC-SIGNR levels in patients with metastatic cervical cancer compared with patients with non-metastatic cervical cancer $(\mathrm{P}=0.1793$; Fig. 4D).

Levels of $S D C$-SIGNR positively correlate with serum levels of SCC-Ag. SCC-Ag serves as a prognostic marker for cervical cancer (26); patients with cervical cancer present with higher levels of serum SCC-Ag compared with healthy individuals (27). Therefore, the association between serum SCC-Ag and sDC-SIGNR levels were analyzed in the group of patients with cervical cancer. Notably, there was a positive correlation between the levels of serum SCC-Ag and SDC-SIGNR $(\mathrm{r}=0.2583$; $\mathrm{P}=0.0348$; Fig. 4E). The levels of sDC-SIGNR were significantly higher in samples with high serum SCC-Ag levels compared with samples with normal serum SCC-Ag levels ( $\mathrm{P}=0.0426$; Fig. 4F).

There is no association between sDC-SIGNR levels and the levels of serum CEA or CAs-199, -153 and -125. Levels of sDC-SIGNR were not associated with age $(r=-0.1172$; $\mathrm{P}=0.2883$; Fig. 5A), and there was no statistically significant difference between sDC-SIGNR levels in patients aged $\geq 50$ and $<50(\mathrm{P}=0.7944$; Fig. 5B). There was no association between sDC-SIGNR levels and the levels of serum CEA $(r=-0.21$; 

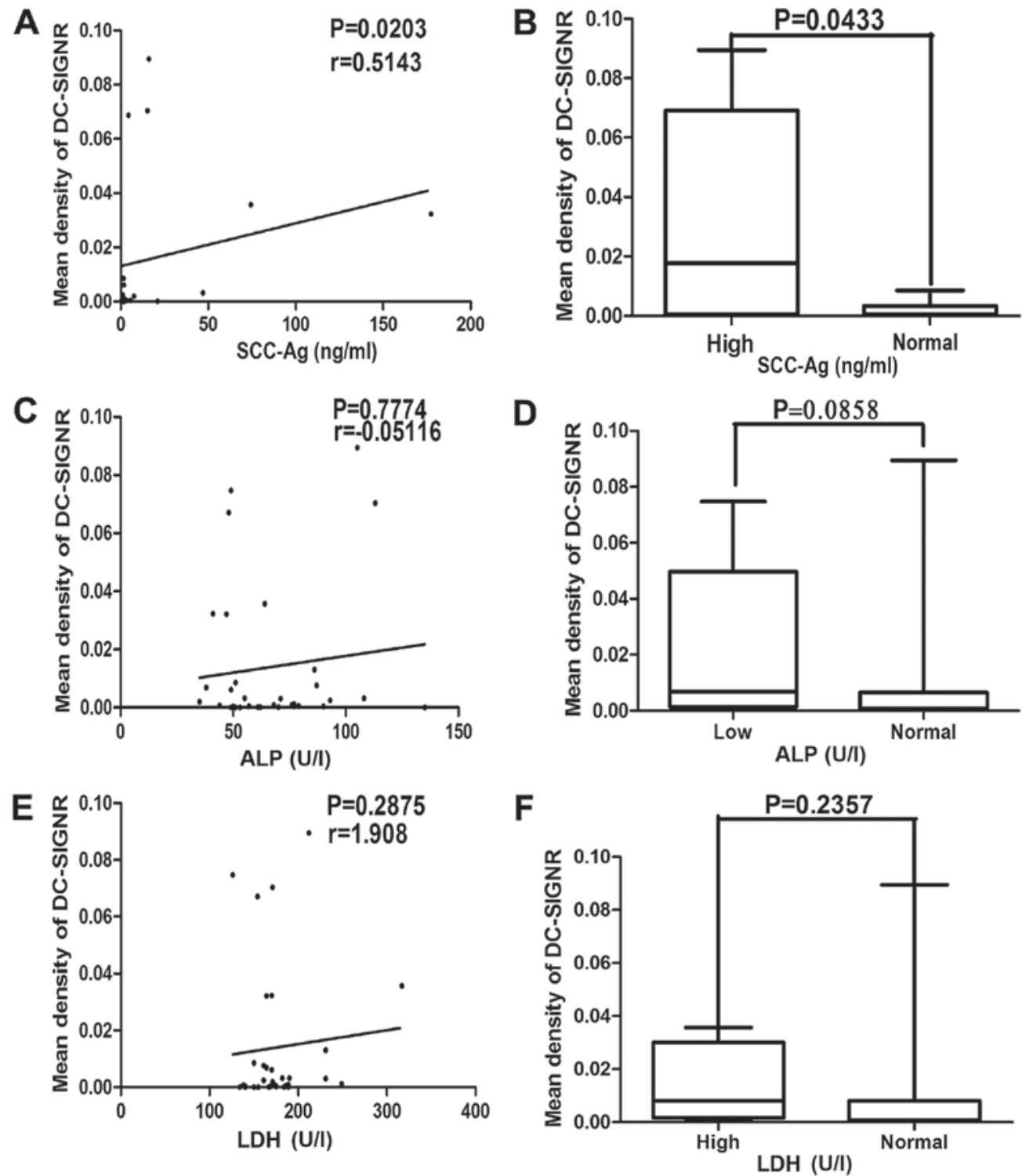

Figure 2. Correlation analysis of DC-SIGNR expression and clinical data. Graphs show (A) the correlation between the mean density of DC-SIGNR from immunohistochemistry analysis and the serum level of SCC-Ag, (B) the mean density of DC-SIGNR in patients with high SCC-Ag serum levels compared with those with normal SCC-Ag levels, (C) the correlation of the mean density of DC-SIGNR and serum ALP levels, (D) the mean density of DC-SIGNR in patients with low ALP levels compared with those with normal ALP levels, (E) the correlation of the mean density of DC-SIGNR and serum LDH levels, (F) the mean density of DC-SIGNR in patients with high LDH levels compared with those with normal LDH levels. Data are presented as the mean \pm standard deviation. DC-SIGNR, dendritic cell-specific intercellular adhesion molecule-3-grabbing nonintegrin-related protein; SCC-Ag, squamous cell carcinoma antigen; ALP, alkaline phosphatase; LDH, lactate dehydrogenase.
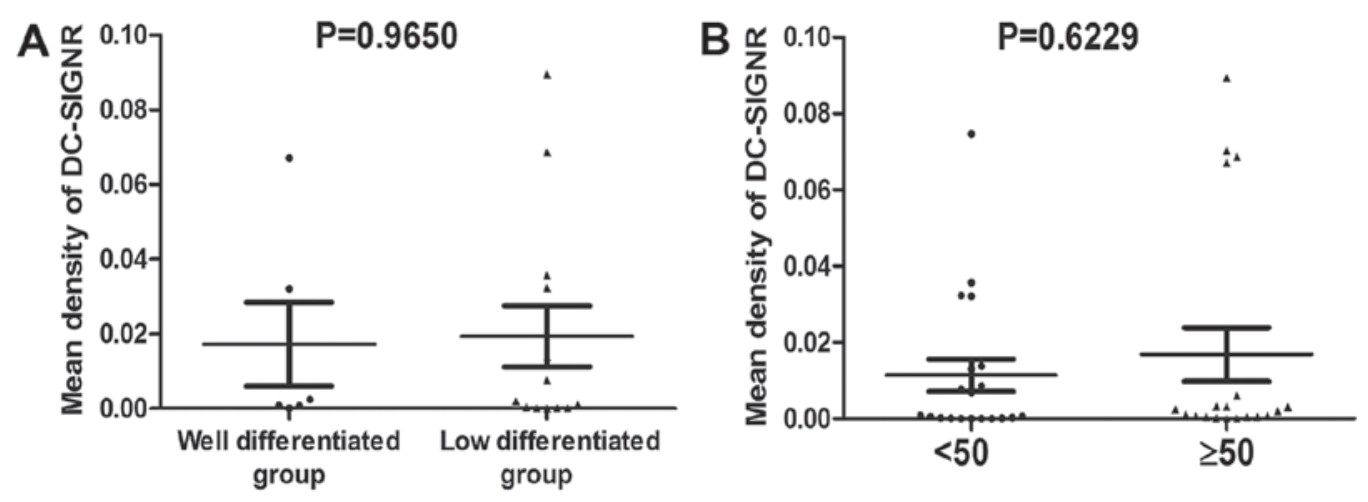

Figure 3. Correlation of DC-SIGNR expression levels with the degree of cervical cancer differentiation and age. (A) The mean density of DC-SIGNR in patients with well differentiated cervical cancer compared with those exhibiting cervical cancer with low differentiation. (B) The mean density of DC-SIGNR in patients $\geq 50$ years old compared with those $<50$ years old. Data are presented as the mean \pm standard deviation. DC-SIGNR, dendritic cell-specific intercellular adhesion molecule-3-grabbing nonintegrin-related protein. 

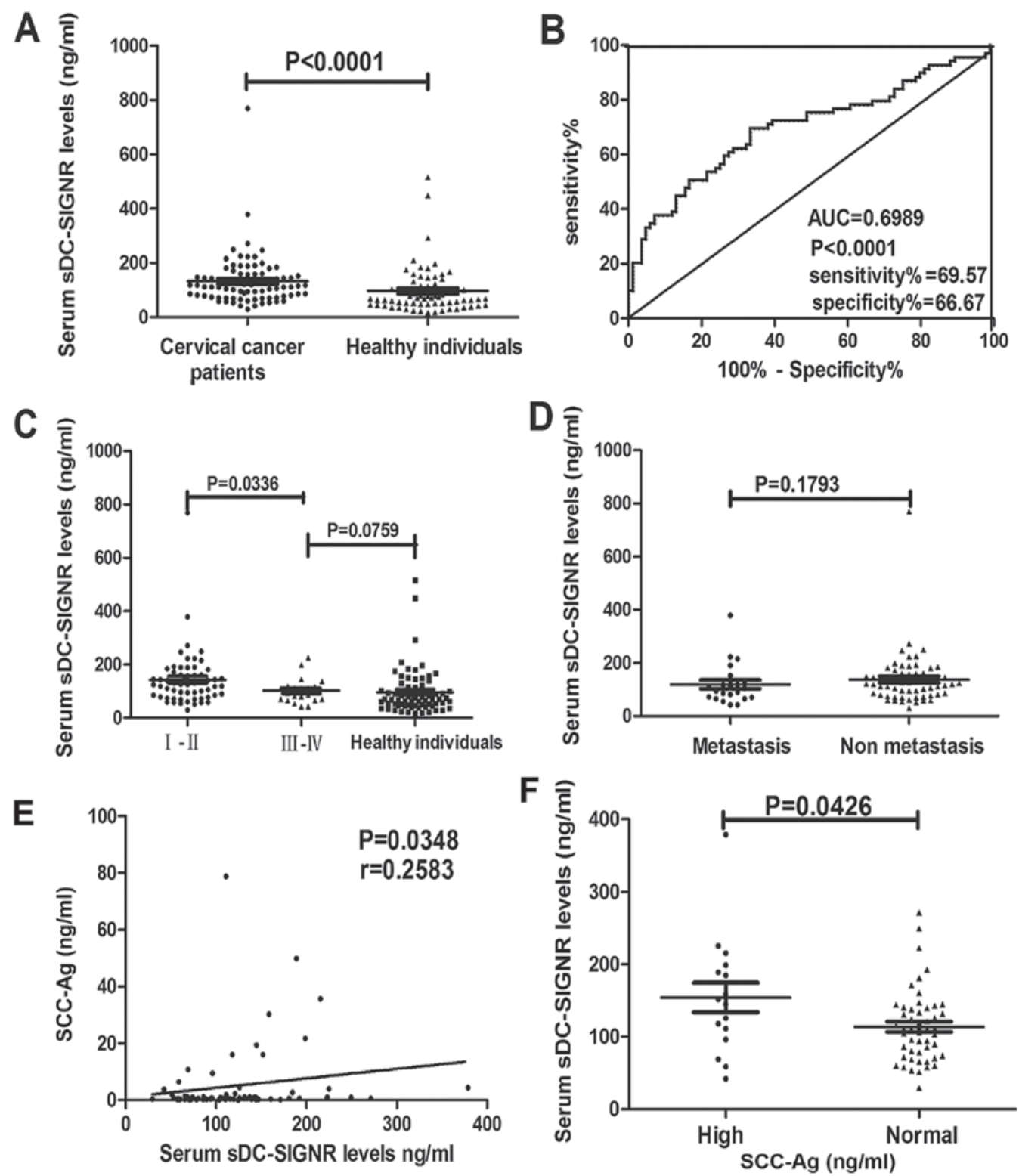

Figure 4. The comparison of sDC-SINGR concentration between different groups of patients with cervical cancer, including the prognostic significance of sDC-SINGR as indicated by the receiver operating characteristic curve, and its association with SCC-Ag. (A) Levels of sDC-SIGNR in 84 patients with cervical cancer compared with that of 69 healthy individuals. (B) Receiver operating characteristic curve analysis was performed to determine the clinical value of sDC-SIGNR levels in cervical cancer prediction. A cut-off value of $93.7 \mathrm{ng} / \mathrm{ml}$ for DC-SIGNR was identified to predict the presence of cervical cancer with $69.57 \%$ sensitivity and $66.67 \%$ specificity (AUC, 0.6989; P<0.0001). (C) Levels of sDC-SIGNR in patients with stage I and II cervical cancer compared with patients with stage III and IV cervical cancer and healthy individuals. (D) Levels of sDC-SIGNR in patients with metastatic and non-metastatic cervical cancer. (E) Correlation between levels of sDC-SIGNR with SCC-Ag in patients with cervical cancer. (F) Levels of serum sDC-SIGNR in patients with cervical cancer with high serum SCC-Ag levels compared with those with normal serum SCC-Ag levels. Data are presented as the mean \pm standard deviation. sDC-SIGNR, serum dendritic cell-specific intercellular adhesion molecule-3-grabbing nonintegrin-related protein; SCC-Ag, squamous cell carcinoma antigen; AUC, area under the curve.

$\mathrm{P}=0.2102$; Fig. 5C), CA-199 (r=-0.2288; P=0.4121; Fig. 5D), CA-153 (r=-0.4978; P=0.0497; Fig. 5E) and CA-125 (r=-0.2035; $\mathrm{P}=0.3087$; Fig. 5F).

\section{Discussion}

DC-SIGNR is a member of the C-type lectin family of proteins. Previous research has focused on the association between DC-SIGNR and viral infections, including human immunodeficiency virus (HIV) $(27,28)$ and hepatitis B (29). Previous studies have indicated that DC-SIGNR is highly expressed in peripheral blood mononuclear cells in patients with HIV-1 infection (30). However the role of DC-SIGNR in cancer, particularly cervical cancer, remains unclear.

In order to study the clinical value of DC-SIGNR in cervical cancer, the mean density of DC-SIGNR in the tissue of 25 patients with cervical cancer, 14 patients with $\mathrm{CIN}$ and 15 patients with cervical polyps was determined by immunohistochemistry. The mean density of DC-SIGNR was significantly higher in cervical cancer tissue compared with $\mathrm{CIN}$ and cervical polyp tissue $(\mathrm{P}=0.0184$ and $\mathrm{P}=0.0236$, respectively). However, there was no significant difference in the mean density between CIN and cervical polyp tissue samples $(\mathrm{P}=0.8103)$. This result suggests that DC-SIGNR 

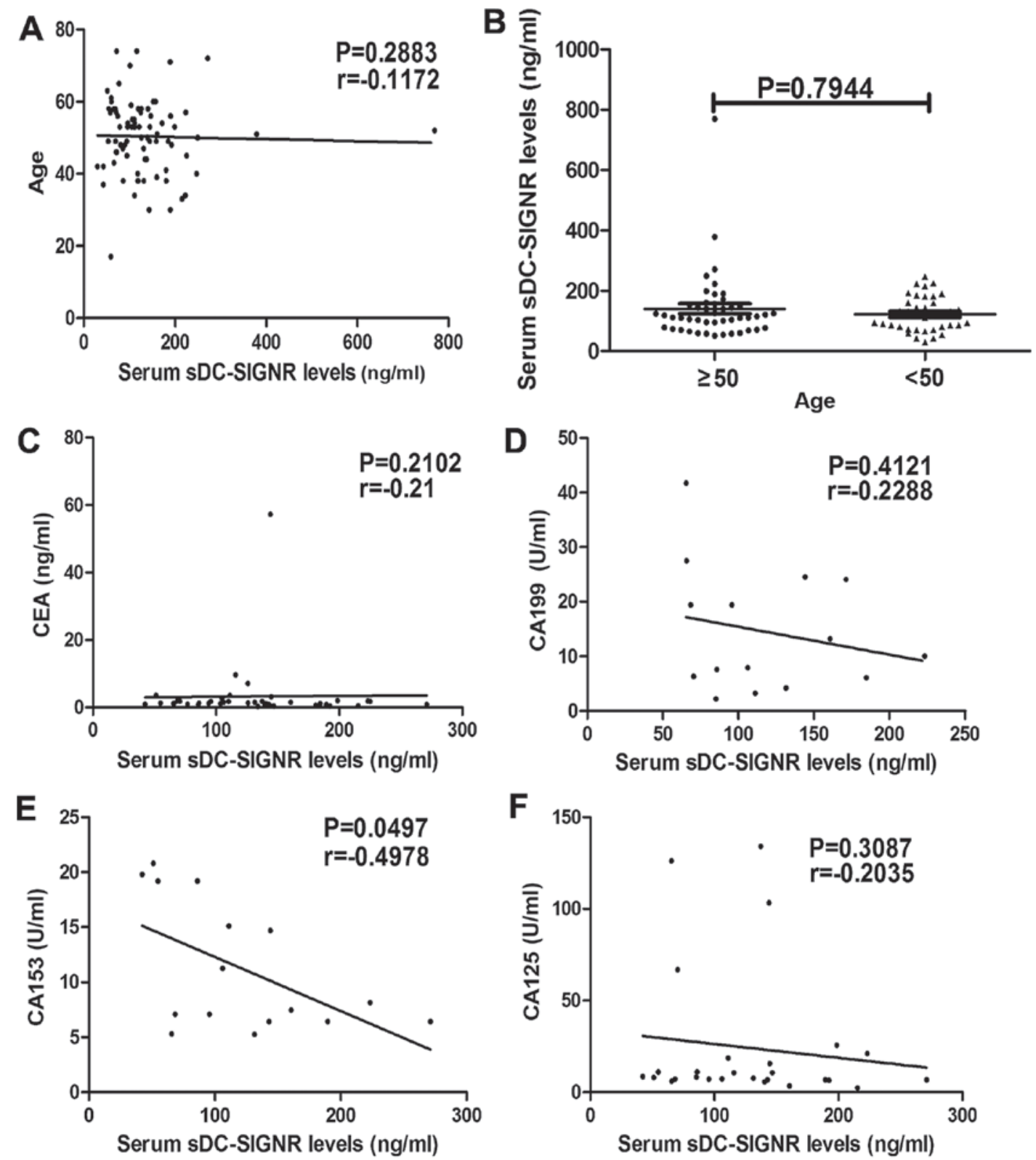

Figure 5. Scatter plots demonstrating the associations between sDC-SIGNR and different clinical factors of patients with cervical cancer. (A) No correlation was identified between the levels of sDC-SIGNR and age. (B) The analysis revealed that there was no significance difference between sDC-SIGNR serum levels in the group of patients $\geq 50$ and those $<50$ years old. No significant correlation was identified between the level of sDC-SIGNR, and (C) CEA, (D) CA199, (E) CA-153 and (F) CA125 in patients with cervical cancer. Data are presented as the mean \pm standard deviation. sDC-SIGNR, serum dendritic cell-specific intercellular adhesion molecule-3-grabbing nonintegrin-related protein; CEA, carcinoembryonic antigen; CA, carbohydrate antigen.

could serve as a biomarker for the early diagnosis of cervical cancer.

The rate of recurrence of cervical cancer in patients is low; however, the sensitivity of the cancer to radiotherapy and chemotherapy is significantly decreased in cases where the cancer is recurrent (31). SCC-Ag is a glycoprotein that belongs to the serpin family of serine/cysteine proteinase inhibitors. SCC-Ag has been associated with the prognosis of recurrent cervical squamous carcinoma (32), and is of great significance in the prognosis and clinical diagnosis of cervical cancer (33-35). In the present study, a correlation between the mean density of DC-SIGNR and the clinical serum SCC-Ag concentration was observed $(r=0.5143 ; P=0.0203)$. However, there was no association between DC-SIGNR and other clinical markers, such as CEA and CA-199, -125 and -153.
Due to the association between DC-SIGNR levels and cervical cancer identified by immunohistochemistry, the levels of sDC-SIGNR in patients with cervical cancer were analyzed by ELISA and compared with the levels of sDC-SIGNR from healthy individuals. The level of sDC-SIGNR was higher in patients with cervical cancer compared with that in healthy individuals, and was significantly positively correlated with SCC-Ag levels. Previous studies have demonstrated that DC-SIGNR expression is lower in patients with NHL and lung cancer compared with that in healthy individuals $(21,36)$; therefore DC-SIGNR may have a clinical application for the diagnosis of a number of types of cancer. sDC-SIGNR levels in patients with stage I and II cervical cancer were significantly higher compared with those in patients with stage III and IV cervical cancer. This result suggests that DC-SIGNR is likely 
to be of most clinical value in the early diagnosis of cervical cancer. There was no significant difference in SDC-SIGNR levels between patients with stage III/IV cervical cancer and healthy individuals. This result may be related to the decline of immune function in the late stages of cancer.

Although pathological diagnosis remains the gold standard for the diagnosis of cervical cancer, serum tumor markers could be important for the early diagnosis of cervical cancer, particularly for high-risk screening. If cancer is detected at an early stage, or even in the pre-malignant stage, physicians will have a higher probability of successfully treating the patient $(37,38)$. The results from the present study indicate that DC-SIGNR and sDC-SIGNR levels could be helpful for the early diagnosis of cervical cancer.

C-type lectins are a large family of proteins that serve roles in several biological processes. Various previous studies have demonstrated that a number of C-type lectins participate in tumor metastasis, for example LSECtin and L-selectin $(39,40)$. Although the specific role DC-SIGNR serves in cervical cancer remains unclear, the results of the present study indicate that DC-SIGNR and sDC-SIGNR levels can be used to distinguish between patients with cervical cancer and healthy individuals, and may allow for the early diagnosis of cervical cancer. Further experiments are required to explore the specific roles DC-SIGNR serves in cervical cancer.

\section{Acknowledgements}

The present study was supported by the Chinese State Key Program in Basic Research (grant no. 2012CB822103), the Chinese National Science Foundation Project (grant nos. 81372669 and 31270867), the Science and Technology Planning Project of Liaoning Province (grant no. 2012225020) and the Chinese Ministry of Health (grant no. W2012RQ23).

\section{References}

1. Parkin DM and Bray F: Chapter 2: The burden of HPV-related cancers. Vaccine 24 (Suppl 3): 3/11-25, 2006.

2. Arbyn M, Castellsagué X, de Sanjosé S, Bruni L, Saraiya M, Bray F and Ferlay J: Worldwide burden of cervical cancer in 2008. Ann Oncol 22: 2675-2686, 2011.

3. World Health Organization: Comprehensive Cervical Cancer Control: A Guide to Essential Practice. 2nd edition. World Health Organization, Geneva, Switzerland, 2014.

4. Mzarico E, Gómez-Roig MD, Guirado L, Lorente N and Gonzalez-Bosquet E: Relationship between smoking, HPV infection, and risk of cervical cancer. Eur J Gynaecol Oncol 36 677-680, 2015

5. Ahmed HG, Bensumaidea SH and Ashankyty IM: Frequency of human papillpoma virus (HPV) subtypes 31,33,35,39 and 45 among Yemeni women with cervical cancer. Infect Aqent Cancer 10: 29, 2015.

6. Hildesheim A and Wanq SS: Host and viral genetics and risk of cervical cancer: A review. Virus Res 89: 229-240, 2002.

7. Luo Q, Zhang S, Wei H, Pang X and Zhang H: Roles of Foxp3 in the occurrence and development of cervical cancer. Int J Clin Exp Pathol 8: 8717-8730, 2015.

8. Dambuza IM and Brown GD: C-type lectins in immunity: Recent developments. Curr Opin Immunol 32: 21-27, 2015.

9. Strenq-Ouwehand I, Unqer WW and Van Kooyk Y: C-type lectin receptors for tumor eradication: Future directions. Cancers (Basel) 3: 3169-3188, 2011.

10. Laskarin G, Redzovic A, Vlastelic I, Haller H, Medancic SS, Solinas G and Rukavina D: Tumor-associated glycoprotein (TAG-72) is a natural ligand for the C-type lectin-like domain that induces anti-inflammatory orientation of early pregnancy decidual $\mathrm{CD} \mathrm{a}^{+}$dendritic cells. J Reprod Immunol 88: 12-23, 2011.
11. Sancho D and Reis e Sousa C: Sensing of cell death by myeloid C-type lectin receptors. Curr Opin Immunol 25: 46-52, 2013.

12. Geijtenbeek TB, van Vliet SJ, Enqering A, 't Hart BA and van Kooyk Y: Self-and nonself-recognition by C-type lectins on dendritic cells. Annu Rev Immunol 22: 33-54, 2004.

13. Soilleux EJ, Barten R and Trowsdale J: DC-SIGN; a related gene, DC-SIGNR; and CD23 from a cluster on 19p13. J Immunol 165: 2937-2942, 2000

14. Martens JH, Kzhyshkowska J, Falkowski-Hansen M, Schledzewski K, Gratchev A, Mansmann U, Schmuttermaier C, Dippel E, Koenen W, Riedel F, et al: Differential expression of a gene signature for scavenger/lectin receptors by endothelial cells and macrophages in human lymph node sinuses, the primary sites of regional metastasis. J Pathol 208: 574-589, 2006.

15. Jianq Y, Zhanq C, Chen K, Chen Z, Sun Z, Zhang Z, Ding D, Ren S and Zuo Y: The clinical significance of DC-SIGN and DC-SIGNR, which are novel markers expressed in human colon cancer. PLoS One 9: e114748, 2014.

16. BIeijs DA, Geijtenbeek TB, Fiqdor CG and van Kooyk Y: DC-SIGN and LFA-1: A battle for ligand. Trends Immunol 22: 457-463, 2001.

17. Lu S, Bevier M, Huhn S, Sainz J, Lascorz J, Pardini B, Naccarati A, Vodickova L, Novotny J, Hemminki K, et al: Genetic variants in C-type lectin genes are associated with colorectal cancer susceptibility and clinical outcome. Int J Cancer 133: 2325-2333, 2013.

18. Ding D, Chen W, Zhang C, Chen Z, Jiang Y, Yang Z, Jiang X, Zuo Y and Ren S: Low expression of dendritic cell-specific intercellular adhesion molecule-3-grabbing nonintegrin in non-Hodgkin lymphoma and a significant correlation with 32-microglobulin. Med Oncol 31: 202, 2014.

19. Liu W, Tang L, Zhang G, Wei H, Cui Y, Guo L, Gou Z, Chen X, Jiang D, Zhu Y, et al: Characterization of a novel C-type lectin-like gene, LSECtin: Demonstration of carbohydrate binding and expression in sinusoidal endothelial cells of liver and lymph node. J Biol Chem 279: 18748-18758, 2004.

20. Pöhlmann S, Soilleux EJ, Baribaud F, Leslie GJ, Morris LS, Trowsdale J, Lee B, Coleman N and Doms RW: DC-SIGNR, a DC-SIGN homologue expression in endothelial cells, binds to human and simian immunodeficiency viruses and activates infection in trans. Proc Natl Acad Sci USA 98: 2670-2675, 2001.

21. Liu X, Zhanq H, Su L, Yang P, Xin Z, Zou J, Ren S and Zuo Y: Low expression of dendritic cell-specific intercellular adhesion molecule-grabbing nonintegrin-related protein in lung cancer and significant correlations with brain metastasis and natural killer cells. Mol Cell Biochem 407: 151-160, 2015.

22. Van de Lande J, Davelaar EM, von Mensdorff-Pouilly S, Water TJ, Berkhof J, van Baal WM, Kenemans P and Verheijen RH: SCC-Ag, lymph node metastases and sentinel node procedure in early stage squamous cell cervical cancer. Gynecol Oncol 112: $119-125,2009$

23. Rauch GM, Kaur H, Choi H, Ernst RD, Klopp AH, Boonsirikamchai P, Westin SN and Marcal LP: Optimization of MR imaging for pretreatment evaluation of patients with endometrial and cervical cancer. Radiographics 34: 1082-1098, 2014.

24. Pöhlmann S, Soilleux EJ, Baribaud F, Leslie GJ, Morris LS, Trowsdale J, Lee B, Coleman N and Doms RW: DC-SIGNR, a DC-SIGN homologue expressed in endothelial cell, binds to human and simian immunodeficiency viruses and activates infection in trans. Proc Natl Acad Sci USA 98: 2670-2675, 2001.

25. Yulin Li (ed): Pathology. 8th edition. People's Medical Publishing House, Beijing, 2013 (In Chinese).

26. Bashirova AA, Geijtenbeek TB, van Duijnhoven GC, van Vliet SJ, Eilering JB, Martin MP, Wu L, Martin TD, Viebig N, Knolle PA, et al: A dendritic cell-specific intercellular adhesion molecule 3-grabbing nonintegrin (DC-SIGN)-related protein is highly expressed on human liver sinusoidal endothelial cells and promotes HIV-1 infection. J Exp Med 193: 671-678, 2001

27. Mitchell DA, Fadden AJ and Drickamer K: A novel mechanism of carbohydrate recognition by the C-type lectins DC-SIGN and DC-SIGNR. Subunit organization and binding to multivalent ligands. J Biol Chem 276: 28939-28945, 2001.

28. Op den Brouw ML, de Jonq MA, Ludwiq IS, van der Molen RG, Janssen HL, Geijtenbeek TB and Woltman AM: Branched oligosaccharide structures on HBV prevent interaction with both DC-SIGN and L-SIGN. J Viral Hepat 15: 675-683, 2008.

29. Soilleux EJ: DC-SIGN (dendritic cell-specific ICAM-grabbing non-integrin) and DC-SIGN-related (DC-SIGNR): Friend or foe? Clin Sci (Lond) 104: 437-446, 2003. 
30. Chaudhary O, Kumar S, Bala M, Singh J, Hazarika A and Luthra K: Association of DC-SIGNR expression in peripheral blood mononuclear cells with DC-SIGNR genotypes in HIV-1 infection. Viral Immunol 28: 472-475, 2015.

31. Jeonq BK, Choi DH, Huh SJ, Park W, Bae DS and Kim BG: The role of squamous cell carcinoma antigen as a prognostic and predictive factor in carcinoma of uterine cervix. Radiat Oncol J 29: 191-198, 2011.

32. Wang Y, Cui T, Du L, Xu X, Tian B, Sun T, Han C, Zhao X and Jing J: The correlation between the serum squamous carcinoma antigen and the prognosis of recurrent cervical squamous carcinoma. J Clin Lab Anal 31, 2017.

33. Shimura K, Mabuchi S, Yokoi T, Sasano T, Sawada K, Hamasaki T and Kimura T: Utility of serum squamous cell carcinoma antigen levels at the time of recurrent cervical cancer diagnosis in determining the opti mal treatment choice. J Gynecol Oncol 24: 321-329, 2013.

34. Kim BG: Squamous cell carcinoma antigen in cervical cancer beyond. J Gynecol Oncol 24: 291-292, 2013.

35. Li X, Zhou J, Huanq K, Tang F, Zhou H, Wang S, Jia Y, Sun H, Ma D and Li S: The predictive value of serum squamous cell carcinoma antigen in patients with cervical who receive neoadjuvant chemotherapy followed by radical surgery: A single-institute study. PLoS One 10: e0122361, 2015.
36. Zhang Z, Chen K, Yan L, Yang Z, Zhu Z, Chen C, Zeng J, Wei W, Qi X, Ren S and Zuo Y: Low expression of dendritic cell-specific intercellular adhesion molecule-grabbing nonintegrin-related protein in non-Hodgkin lymphoma and significant correlations with lactic dehydrogenase and $\beta 2$-microglobulin. Biochem Cell Biol 91: 214-220, 2013.

37. Wulfkuhle JD, Liotta LA and Petricoin EF: Proteomic applications for the early detection of cancer. Nat Rev Cancer 3: 267-275, 2003.

38. Gdowski A, Ranjan AP, Mukerjee A and Vishwanatha JK: Nanobiosensors: Role in cancer detection and diagnosis. Adv Exp Med Biol 807: 33-58, 2014.

39. Zuo Y, Ren S, Wanq M and Liu B, Yang J, Kuai X, Lin C, Zhao D, Tang $\mathrm{L}$ and He F: Novel roles of liver sinusoidal endothelial cell lectin in colon carcinoma cell adhesion, migration and in-vivo metastasis to the liver. Gut 62: 1169-1678, 2013.

40. Qian F, Hanahan D and Weissman IL: L-selectin can facilitate metastasis to lymph nodes in a transgenic mouse model of carcinogenesis. Proc Natl Acad Sci USA 98: 3976-3981, 2001. 